\title{
A Robust Peak-to-Average Power Ratio Reduction Scheme by Inserting Dummy Signals with Enhanced Partial Transmit Sequence in OFDM Systems.
}

\begin{abstract}
Peak-to-average power ratio (PAPR) is one of the main drawbacks in orthogonal frequency division multiplexing (OFDM) systems. High PAPR forces the power amplifier to back off in order to operate in its linear region, which degrades the power efficiency of the system. Several PAPR reduction techniques have been developed, but most of them have not considered both complexity and PAPR reduction. In this paper, a novel PAPR reduction scheme based on the insertion of dummy sequences to an enhanced partial transmit sequence is proposed. By applying this scheme the PAPR performance is enhanced compared to the conventional methods while the complexity is significantly reduced. Numerical analysis is carried out with OFDM signal and QPSK modulation.
\end{abstract}

Keyword: Power amplifier; Orthogonal frequency division multiplexing; Partial transmit sequence; PAPR; Dummy signals 